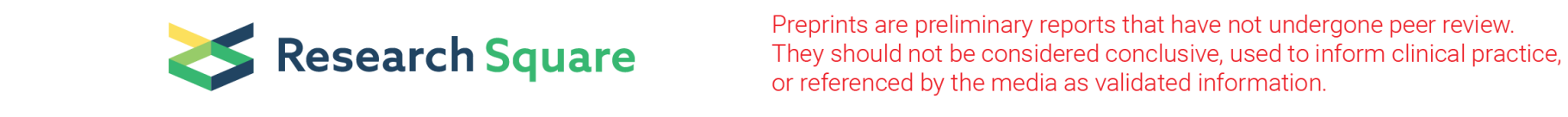

\title{
Mining image data to better characterize cancer
}

Chintan Parmar

Ralph T. H. Leijenaar

Patrick Grossmann

Emmanuel Rios Velazquez

Johan Bussink

Derek Rietveld

Michelle M. Rietbergen

Benjamin Haibe-Kains

Philippe Lambin

Hugo J.W.L. Aerts

\section{Video Abstract}

Keywords: Biomarkers, Cancer, Consensus clustering, CT, Diagnosis, H\&amp;N cancer, Head and neck cancer, Image features, Imaging, Lung cancer, Medical, Oncology, Patient, Precision medicine, Radiology, Radiomics, Scientific Reports, Treatment, Tumor phenotypes, X-ray computed tomography

Posted Date: September 20th, 2019

DOl: https://doi.org/10.21203/rs.2.15110/v1

License: (c) (i) This work is licensed under a Creative Commons Attribution 4.0 International License. Read Full License 


\section{Abstract}

Whether it be MRI, CT, or PET scans, nearly every cancer patient undergoes some form of imaging. These images provide important information about the location and stage of tumor growth and can, therefore, inform treatment strategies. But recent advances in Artificial Intelligence make it possible to mine these images for even more data. This emerging field, called 'radiomics' aims to utilize the full potential of medical images by extracting high-dimensional data to objectively characterize and monitor individual tumors. Many current cancer-detection and diagnostic techniques rely on invasive approaches such as tissue biopsies. But these practices have both high risk and high cost. Radiomics, on the other hand, offers a promising method to gather important information about tumors - such as size, shape, and texture -- in a non-invasive and often cost-effective manner. To provide further insights into the utility of this approach, a study published in Scientific Reports extracted cancer-specific signatures from CT images of patients with lung and head-and-neck cancers. The research team applied consensus clustering of over four-hundred image feature algorithms. This resulted in approximately a dozen clusters for each cancer type. Statistical analyses indicated these clusters were significantly associated with patient survival and tumor stage. They also found that analyzing image signatures allowed them to predict the likely progression of the disease with moderate to high accuracy. Radiomics has the potential to greatly enhance patient care by providing physicians a means to assess the physical and molecular characteristics of a tumor without the need for painful retrieval of a tissue sample. The fact that images are routinely obtained for cancer diagnosis anyway, makes this a cost-effective approach. Additionally, because biopsies are often taken only once and in the early stages following detection, they cannot fully capture the progression or treatment-response of the disease. A radiomic approach can do both. 